\title{
Magnetic Resonance Imaging Texture of Medial Pulvinar in Dementia with Lewy Bodies
}

\author{
Kayeong Tak ${ }^{a}$ Subin Lee ${ }^{a}$ Euna Choi ${ }^{b}$ Seung Wan Suh ${ }^{c}$ Dae Jong Oh ${ }^{d}$ \\ Woori Moon $^{d}$ Hye Sung Kim ${ }^{d}$ Seonjeong Byun ${ }^{d}$ Jong Bin Bae ${ }^{d}$ Ji Won Han ${ }^{d}$ \\ Jae Hyoung Kim ${ }^{e}$ Ki Woong Kim ${ }^{a}$,,$f$ \\ ${ }^{a}$ Department of Brain and Cognitive Sciences, Seoul National University College of Natural Sciences, \\ Seoul, Republic of Korea; ${ }^{b}$ National Institute of Dementia, Seongnam, Republic of Korea; ${ }^{c}$ Department of \\ Psychiatry, Kangdong Sacred Heart Hospital, Hallym University College of Medicine, Seoul, Republic of Korea; \\ ${ }^{\mathrm{d}}$ Department of Neuropsychiatry, Seoul National University Bundang Hospital, Seongnam, Republic of Korea; \\ e Department of Radiology, Seoul National University Bundang Hospital, Seongnam, Republic of Korea; \\ ${ }^{f}$ Department of Psychiatry, Seoul National University College of Medicine, Seoul, Republic of Korea
}

\section{Keywords}

Dementia with Lewy bodies · Pulvinar nuclei · Texture analysis · Gray-level co-occurrence matrix

\begin{abstract}
Introduction: Executive dysfunction is common in dementia with Lewy bodies (DLB). The pulvinar nucleus plays a role in executive control and synchronizes with cortical regions in the salience network that are vulnerable to Lewy pathology. Objective: We investigated the pulvinar subregions in patients with mild DLB and their associations with executive function. Methods: The sample consisted of 38 DLB patients and 38 age- and sex-matched normal controls. We evaluated cognitive function using the Consortium to Establish a Registry for Alzheimer's Disease Assessment Packet. We obtained four pulvinar nuclei using preprocessed T1-weighted magnetic resonance images. We compared volumes and textures of the DLB patients and the normal controls for each nucleus. We used a linear regression to determine the association of textures and neuropsychological test scores. $\boldsymbol{R e}$ -
\end{abstract}

(c) 2020 S. Karger AG, Basel

\section{karger@karger.com}

www.karger.com/dem sults: The DLB patients showed comparable volumes to the normal controls in all pulvinar nuclei. However, the DLB patients showed different texture of the left medial pulvinar (PuM) from the normal controls. The entropy, contrast, and cluster shade were lower but autocorrelation of left PuM was higher in the DLB patients compared to the normal controls. These texture features of the left PuM were associated with the set-shifting performance measured by the Trail Making Test. Conclusions: In DLB, the left PuM may be altered from early stage, which may contribute to the development of executive dysfunction.

(c) 2020 S. Karger AG, Basel

\section{Introduction}

Dementia with Lewy bodies (DLB) is the second most common neurodegenerative dementia after Alzheimer's disease. Lewy body pathology results from alpha-synuclein aggregation in nerve cells of the neocortical, limbic, and brainstem regions $[1,2]$. DLB is clinically character- 
ized by progressive cognitive decline in addition to core clinical features, such as fluctuating cognition, recurrent visual hallucinations, and parkinsonism [2]. In the mild stage, DLB patients had more rapid cognitive decline than Alzheimer's disease patients [3]. The cognitive impairment is more likely associated with nonamnestic domains, such as attention and executive function $[4,5]$. Executive dysfunction may be caused by atrophy of the insular and anterior cingulate cortices in the early stage of DLB $[6,7]$. The early vulnerable regions compose the salience network (SN) that initiates cognitive control as it modulates the switch between default mode network and central executive network [8]. Since connectivity within the $\mathrm{SN}$ decreased in DLB patients [9], executive function can be declined.

The pulvinar of the thalamus is divided into 4 nuclei: anterior pulvinar, medial pulvinar (PuM), lateral pulvinar, and inferior pulvinar [10]. Although the pulvinar was commonly referred to as the visual pulvinar [11], the structure also contributed to executive function [12]. In particular, the PuM has widespread connections with the prefrontal cortex and association areas, including the vulnerable regions in SN [13-15]. Previous studies using functional magnetic resonance imaging (MRI) found that the PuM mirrors SN connectivity in other diseases $[16,17]$. Moreover, in recent postmortem studies on DLB patients, Lewy body pathology was present throughout the pulvinar but was most severe in the PuM [18]. However, the volume of anteromedial pulvinar was comparable between DLB patients and the normal controls [18].

Until now, there were no structural or functional neuroimaging studies on the pulvinar nucleus of DLB patients in vivo. Since the volume of pulvinar did not reflect the Lewy pathology of PuM in a postmortem study, the volumetric study on brain MRI may not detect the neuronal degeneration of pulvinar in DLB patients. In contrast to the volumetric measures, the texture measures can identify changes at the neuronal level on brain MRI, because gray matter neuronal density was inversely associated with the T1 relaxation time [19]. Neuronal losses decrease macromolecules and increase extracellular water content, and thus, increase the $\mathrm{T} 1$ relaxation time and reduce the signal intensity on MRI. Neuronal destruction, as well as neuronal loss, also decrease the signal intensity on MRI [20]. To investigate the possible changes of the PuM and their association with executive dysfunction in DLB, we examined the changes in the volume and texture of the pulvinar nuclei and their association with neuropsychological test performance.

\section{Methods}

\section{Subjects}

We recruited 38 DLB patients from the dementia clinic of Seoul National University Bundang Hospital. We enrolled 38 age- and sex-matched cognitively normal controls from the participants of the Korean Longitudinal Study on Cognitive Aging and Dementia (KLOSCAD), which is a nationwide population-based prospective cohort study on cognitive aging and dementia launched in 2009 [21]. The characteristics of the subjects are summarized in Table 1. All patients (or their guardians) and control subjects were fully informed about the study, and written consent was obtained from them. The study was approved by the Institutional Review Board of the Seoul National University Bundang Hospital, Republic of Korea.

\section{Assessments}

Geriatric neuropsychiatrists performed a standardized diagnostic interview, physical and neurological examinations, and laboratory tests for all participants using the Korean version of the Mini International Neuropsychiatric Interview [22] and the Consortium to Establish a Registry for Alzheimer's Disease Assessment Packet (CERAD-K) Clinical Assessment Battery [23]. Trained research neuropsychologists or nurses evaluated global cognitive function using the Mini-Mental State Examination (MMSE) to ascertain the presence of cognitive impairment. We diagnosed DLB based on the consensus guidelines proposed by McKeith et al. [2] and determined the global severity of dementia by the Clinical Dementia Rating [24]. We performed the digit span (forward and backward) by scoring 1 or 0 (pass or fail) to repeat the sequence, and the Trail Making Test (TMT-A and TMT-B) by measuring time to connect distributed numbers or numbers and letters alternately in ascending order $[23,25]$.

\section{Neuroimaging Analysis}

All subjects underwent three-dimensional structural T1weighted spoiled gradient echo MRI scanning on a 3.0 Tesla GE SIGNA scanner (GE Healthcare, Milwaukee, WI, USA) within one and a half years from the clinical assessments. The MRI images were obtained based on the following parameters: voxel size of 1.0 $\times 0.5 \times 0.5 \mathrm{~mm}^{3}, 1.0 \mathrm{~mm}$ sagittal slice thickness with no inter-slice gap, echo time of $3.68 \mathrm{~ms}$, repetition time of $25.0 \mathrm{~ms}$, number of excitations of 1 , flip angle of $90^{\circ}$, field of view of $240 \times 240 \mathrm{~mm}$, and $175 \times 240 \times 240$ matrix in the $x, y$, and $z$ dimensions. To exclude subjects with the risk of concomitant vascular pathology, T2-weighted FLAIR MRI was obtained with the 3.0 Tesla scanner system (GE Healthcare, Milwaukee, WI, USA) using the following protocols: voxel size of $0.5 \times 0.5 \times 3.0 \mathrm{~mm}^{3}, 3.0 \mathrm{~mm}$ axial slice thickness, echo time of $160 \mathrm{~ms}$, repetition time of $9,900 \mathrm{~ms}$, number of excitations of 1 , flip angle of $90^{\circ}$, inversion time of $2,500 \mathrm{~ms}$, field of view of $240 \times 240 \mathrm{~mm}$, and $256 \times 256$ matrix in axial plane.

We used the original Digital Imaging and Communications in Medicine (DICOM) format images, and converted to NIfTI format for analysis using MRIcron software. We resliced the images to isovoxels $\left(1.0 \times 1.0 \times 1.0 \mathrm{~mm}^{3}\right)$. Then we automatically segmented whole brain structures by recon-all streams of the FreeSurfer development version 7.0 (http://surfer.nmr.mgh.harvard. edu) [26]. We segmented the thalamus automatically from the preprocessed T1 MRI images using the FreeSurfer software. For each subject, we developed the masks of 26 different nuclei, each in both 
Table 1. Demographic and clinical characteristics of the participants

\begin{tabular}{|c|c|c|c|}
\hline & $\operatorname{DLB}(n=38)$ & $\mathrm{NC}(n=38)$ & $p^{*}$ \\
\hline Age, years & $74.89 \pm 6.30$ & $74.84 \pm 6.15$ & 0.971 \\
\hline Sex (men) & $19(50.00)$ & $19(50.00)$ & 1.000 \\
\hline Educational level, years & $11.00 \pm 5.24$ & $11.45 \pm 3.98$ & 0.832 \\
\hline MMSE, points & $19.29 \pm 5.43$ & $27.47 \pm 1.93$ & $<0.001$ \\
\hline Clinical Dementia Rating & & & $<0.001$ \\
\hline 0 & 0 & $38(100.00)$ & \\
\hline 0.5 & $27(0.71)$ & 0 & \\
\hline 1 & $9(0.24)$ & 0 & \\
\hline 2 & $2(0.05)$ & 0 & \\
\hline 3 & 0 & 0 & \\
\hline \multicolumn{4}{|l|}{ Digit span, points } \\
\hline Forward & $4.38 \pm 1.69$ & $7.14 \pm 2.57$ & $<0.001$ \\
\hline Backward & $3.00 \pm 1.35$ & $5.20 \pm 1.37$ & $<0.001$ \\
\hline \multicolumn{4}{|l|}{ Trail Making Test, s } \\
\hline A & $189.19 \pm 116.21$ & $58.26 \pm 26.21$ & $<0.001$ \\
\hline $\mathrm{B}$ & $322.94 \pm 60.15$ & $181.13 \pm 89.52$ & $<0.001$ \\
\hline Diff & $133.75 \pm 98.10$ & $122.87 \pm 75.54$ & 0.544 \\
\hline eTIV, $\mathrm{mm}^{3}$ & $1,596,580.50 \pm 167,542.68$ & $1,565,892.06 \pm 192,721.44$ & 0.461 \\
\hline
\end{tabular}

Data presented as mean \pm SD or $n(\%)$. DLB, dementia with Lewy bodies; NC, normal controls; MMSE, MiniMental State Examination; Trail Making Test-Diff, Trail Making Test-B minus Trail Making Test-A; eTIV, estimated total intracranial volume * Student $t$ test, independent-samples Mann-Whitney U test, or chi-square test.

the left and right thalamus, defined by a probabilistic atlas developed with histological data [27]. Among the 26 nuclei, we analyzed the left and right side of the anterior pulvinar, PuM, lateral pulvinar, and inferior pulvinar in the current study.

We measured the volumes of pulvinar subregions and estimated total intracranial volumes (eTIV) using the FreeSurfer. We analyzed the four texture features (entropy, contrast, autocorrelation, and cluster shade) of each pulvinar nucleus from T1-weighted MRI images using the gray-level co-occurrence matrices (GLCMs). The GLCM is an N (number of gray levels) square matrix. Each element $(i, j)$ of the matrix reports how many times specific pairs of gray level values, including a reference voxel $\mathrm{i}$ and a neighboring voxel $\mathrm{j}$, occur at distance $\mathrm{d}$ and direction $\otimes$. Possible combinations of $d$ and $\nabla$ make the GLCMs, while the matrices are averaged to obtain a final GLCM, and the texture features are calculated from the final GLCM [28]. For histogram normalization of each pulvinar nucleus, voxels with intensities ranging between $[\mu-3 \sigma]$ and $[\mu+3 \sigma]$ were included to avoid infrequent intensity values; $\mu$ is the mean value of the gray levels and $\sigma$ is the standard deviation [29]. Then we quantized gray levels in $8 \times 8$ matrices to minimize zero valued entries [30]. For the quantization, the 3D GLCMs were generated with a distance of $\mathrm{d}=1$ between voxel pairs and in 13 independent directions [31]. Based on averaged 13 GLCMs, we calculated four texture features in each region. The researcher was not blinded to the diagnosis when she analyzed the GLCMs. We performed the texture analysis using the MATLAB R2014b (Mathworks, Natick, MA, USA).

\section{Statistical Analysis}

We performed descriptive statistics to understand the demographic and clinical data of the two groups. We derived TMT-Diff, which is TMT-B minus TMT-A. We used independent-samples $t$ test for age and eTIV, and chi-square test for sex, Clinical Dementia Rating, and digit span backward. Educational level, MMSE scores, digit span forward, and TMT did not follow a normal distribution in normality tests (Kolmogorov-Smirnov or ShapiroWilk). Then, we compared these variables with independent-samples Mann-Whitney U test. The digit span test scores were available for 35 normal controls and 37 DLB, and TMT scores were available for 36 out of 38 DLB patients.

We compared volumes of pulvinar subregions between the DLB group and the normal control group to detect whether atrophy progressed in the patient groups. We performed one-way analysis of covariance (ANCOVA), controlling potential confounding effects of age, sex, total years of education, and eTIV. For non-normally distributed data in right lateral pulvinar, we conducted independent-samples Mann-Whitney U test. In each nucleus, we compared texture feature values among the groups via independent-samples $t$ test or independent-samples Mann-Whitney $U$ test to evaluate gray level distribution.

We performed a linear regression analysis, entering the texture feature values that were different between the DLB patients and the normal controls as independent variables and the neuropsychological test scores as dependent variables. For the data sets that violated normal distribution of residuals, we used log-transformed neuropsychological test scores as dependent variables.
10

Dement Geriatr Cogn Disord 2020;49:8-15 DOI: $10.1159 / 000506798$
Tak/Lee/Choi/Suh/Oh/Moon/Kim/Byun/ $\mathrm{Bae} / \mathrm{Han} / \mathrm{Kim} / \mathrm{Kim}$ 
Table 2. Volumes $\left(\mathrm{mm}^{3}\right)$ of pulvinar subregions

\begin{tabular}{llll}
\hline Subregion & DLB $(n=38)$ & NC $(n=38)$ & $p^{*}$ \\
\hline Left anterior pulvinar & $196.47 \pm 28.10$ & $203.15 \pm 34.95$ & 0.132 \\
Right anterior pulvinar & $164.48 \pm 21.59$ & $172.09 \pm 23.12$ & 0.054 \\
Left medial pulvinar & $917.76 \pm 135.68$ & $931.45 \pm 137.51$ & 0.304 \\
Right medial pulvinar & $760.98 \pm 93.68$ & $786.80 \pm 110.16$ & 0.121 \\
Left lateral pulvinar & $213.14 \pm 54.23$ & $198.11 \pm 37.21$ & 0.249 \\
Right lateral pulvinar & $155.58 \pm 22.84$ & $157.22 \pm 36.19$ & 0.983 \\
Left inferior pulvinar & $192.92 \pm 36.11$ & $191.80 \pm 28.34$ & 0.741 \\
Right inferior pulvinar & $159.84 \pm 25.77$ & $155.62 \pm 27.84$ & 0.637 \\
\hline
\end{tabular}

Data presented as mean \pm SD. * One-way analysis of covariance, adjusting confounding factors (age, gender, educational years, and eTIV), and independent-samples Mann-Whitney U test for right lateral pulvinar.

We performed all statistical analyses with the Statistical Package for the Social Sciences (SPSS) version 20.0 (IBM Corporation, Armonk, NY, USA) on Windows. A $p$ value less than 0.05 was considered statistically significant in the analyses.

\section{Results}

As summarized in Table 1, DLB patients were comparable to normal controls in age, sex, and educational level. Although DLB patients showed lower MMSE scores than the normal controls, their eTIV were comparable to those of the normal controls. The DLB patients presented lower digit span test scores and longer time to perform the TMT than the normal controls did.

The volumes of the pulvinar and the pulvinar subregions were comparable between DLB patients and the normal controls (Table 2). The texture of the pulvinar was also comparable between the two groups. However, when the texture of the pulvinar subregions were compared separately, DLB patients presented lower entropy, contrast, and cluster shade than the normal controls, but higher autocorrelation than the normal controls in the left PuM (Table 3). For linear regression analysis with all participants, texture features of left PuM except autocorrelation were positively associated with the performance of MMSE, while autocorrelation was negatively associated with MMSE. Entropy and contrast were positively associated with log-transformed digit span forward and digit span backward and were negatively associated with $\log$ TMT (A, B, and Diff). In particular, as shown in Figure 1, the texture features had relatively high correlations with log TMT-A (entropy: adjusted $R^{2}=0.231, \beta=-0.492, p<0.001$ and contrast: adjusted $\left.R^{2}=0.281, \beta=-0.539, p<0.001\right)$ and with log TMT-
B (entropy: adjusted $R^{2}=0.226, \beta=-0.487, p<0.001$ and contrast: adjusted $\left.R^{2}=0.290, \beta=-0.547, p<0.001\right)$. Furthermore, for linear regression analysis with DLB, TMTDiff was positively associated with autocorrelation (adjusted $R^{2}=0.172, \beta=0.443, p=0.007$ ) and negatively associated with cluster shade (adjusted $R^{2}=0.105, \beta=-0.361$, $p=0.030$ ) (Fig. 2). However, none of the texture features were associated with the performance of MMSE, digit span test, TMT-A, and TMT-B $(p>0.05)$.

\section{Discussion}

The volumes of the pulvinar nuclei were comparable between the DLB patients and the normal controls, which is in line with a previous study by Erskine et al. [18]. They reported that the volume of anteromedial pulvinar tissue was not affected by Lewy pathology. However, this study found that the texture of left PuM was different between the DLB patients and the normal controls and associated with executive control.

The PuM consists of small, dispersed, and pale neurons [32]. We found that DLB patients presented lower entropy, contrast, and cluster shade than the normal controls, but higher autocorrelation than the normal controls (Table 3). Lower entropy value indicates orderliness of gray level distribution, and smaller contrast value means uniformity of local intensity values among neighboring voxels in an image [33, 34]; meanwhile, lower cluster shade value indicates that the GLCM is skewed to the left in a symmetric image $[28,33,34]$. Therefore, the DLB patients may have smoother, softer, and more repetitive $\mathrm{PuM}$ textures than the normal controls. Previous studies have suggested that the PuM can be affected by Lewy 
Table 3. Texture features for pulvinar nuclei

\begin{tabular}{|c|c|c|c|}
\hline Texture feature & $\mathrm{DLB}(n=38)$ & $\mathrm{NC}(n=38)$ & $p^{*}$ \\
\hline \multicolumn{4}{|l|}{ Left anterior pulvinar } \\
\hline Entropy & $3.17 \pm 0.15$ & $3.18 \pm 0.17$ & 0.506 \\
\hline Contrast & $2.76 \pm 0.57$ & $2.81 \pm 0.58$ & 0.672 \\
\hline Autocorrelation & $18.47 \pm 3.00$ & $18.09 \pm 3.37$ & 0.611 \\
\hline Cluster shade & $-0.94 \pm 2.88$ & $-1.37 \pm 2.94$ & 0.524 \\
\hline \multicolumn{4}{|c|}{ Right anterior pulvinar } \\
\hline Entropy & $3.14 \pm 0.14$ & $3.16 \pm 0.18$ & 0.528 \\
\hline Contrast & $2.93 \pm 0.59$ & $2.82 \pm 0.63$ & 0.424 \\
\hline Autocorrelation & $17.66 \pm 2.27$ & $18.69 \pm 3.00$ & 0.095 \\
\hline Cluster shade & $-0.13 \pm 2.25$ & $-0.92 \pm 3.00$ & 0.085 \\
\hline \multicolumn{4}{|l|}{ Left medial pulvinar } \\
\hline Entropy & $2.93 \pm 0.12$ & $3.09 \pm 0.16$ & $<0.001$ \\
\hline Contrast & $1.91 \pm 0.25$ & $2.48 \pm 0.58$ & $<0.001$ \\
\hline Autocorrelation & $18.91 \pm 1.74$ & $17.38 \pm 2.85$ & 0.006 \\
\hline Cluster shade & $-2.13 \pm 1.95$ & $0.16 \pm 3.45$ & $<0.001$ \\
\hline \multicolumn{4}{|l|}{ Right medial pulvinar } \\
\hline Entropy & $2.96 \pm 0.09$ & $2.97 \pm 0.11$ & 0.662 \\
\hline Contrast & $1.92 \pm 0.20$ & $1.92 \pm 0.23$ & 0.979 \\
\hline Autocorrelation & $19.63 \pm 2.17$ & $19.38 \pm 2.04$ & 0.648 \\
\hline Cluster shade & $-1.89 \pm 2.03$ & $-1.78 \pm 2.20$ & 0.828 \\
\hline \multicolumn{4}{|l|}{ Left lateral pulvinar } \\
\hline Entropy & $3.11 \pm 0.15$ & $3.10 \pm 0.16$ & 0.679 \\
\hline Contrast & $2.57 \pm 0.43$ & $2.51 \pm 0.56$ & 0.588 \\
\hline Autocorrelation & $16.33 \pm 2.75$ & $17.26 \pm 2.91$ & 0.155 \\
\hline Cluster shade & $0.86 \pm 2.58$ & $0.35 \pm 3.52$ & 0.470 \\
\hline \multicolumn{4}{|l|}{ Right lateral pulvinar } \\
\hline Entropy & $3.07 \pm 0.19$ & $3.06 \pm 0.21$ & 0.947 \\
\hline Contrast & $2.67 \pm 0.49$ & $2.57 \pm 0.60$ & 0.437 \\
\hline Autocorrelation & $17.42 \pm 3.00$ & $17.68 \pm 2.80$ & 0.694 \\
\hline Cluster shade & $1.51 \pm 2.88$ & $1.32 \pm 3.31$ & 0.789 \\
\hline \multicolumn{4}{|l|}{ Left inferior pulvinar } \\
\hline Entropy & $3.01 \pm 0.19$ & $3.04 \pm 0.18$ & 0.390 \\
\hline Contrast & $2.50 \pm 0.59$ & $2.51 \pm 0.57$ & 0.947 \\
\hline Autocorrelation & $19.33 \pm 2.49$ & $18.42 \pm 2.40$ & 0.112 \\
\hline Cluster shade & $-0.31 \pm 1.64$ & $-0.28 \pm 1.74$ & 0.949 \\
\hline \multicolumn{4}{|l|}{ Right inferior pulvinar } \\
\hline Entropy & $2.88 \pm 0.24$ & $2.90 \pm 0.27$ & 0.726 \\
\hline Contrast & $2.29 \pm 0.57$ & $2.30 \pm 0.59$ & 0.928 \\
\hline Autocorrelation & $18.61 \pm 3.12$ & $19.05 \pm 3.10$ & 0.542 \\
\hline Cluster shade & $0.19 \pm 2.52$ & $-0.16 \pm 2.38$ & 0.542 \\
\hline
\end{tabular}

Values presented as mean \pm SD. ${ }^{*}$ Student $t$ test or independent-samples Mann-Whitney U test.

body pathology. According to Erskine et al. [18], alphasynuclein was most heavily deposited in the PuM among the pulvinar nuclei. Furthermore, the PuM interconnects with widespread cerebral cortical regions, such as the frontal cortices, including the orbitofrontal cortex and the dorsolateral prefrontal cortex. The PuM also interconnects with the parietotemporal association areas, including the superior temporal area, inferior temporal area, and inferior parietal area, as well as the cingulate and insular cortex $[13,14,35]$, which are highly affected by Lewy body pathology in DLB [36]. According to cell-tocell propagation, alpha synuclein oligomers spread to neurons through anatomically connected networks [37]. Alpha synuclein oligomers, which are neurotoxic [38] and exacerbate synaptic degeneration [39], may spread to interconnected $\mathrm{PuM}$ from neocortical regions and lead to deficient synaptic transmission, as verified in the hippocampus [40]. The texture changes lateralized to the left
Tak/Lee/Choi/Suh/Oh/Moon/Kim/Byun/ $\mathrm{Bae} / \mathrm{Han} / \mathrm{Kim} / \mathrm{Kim}$ 


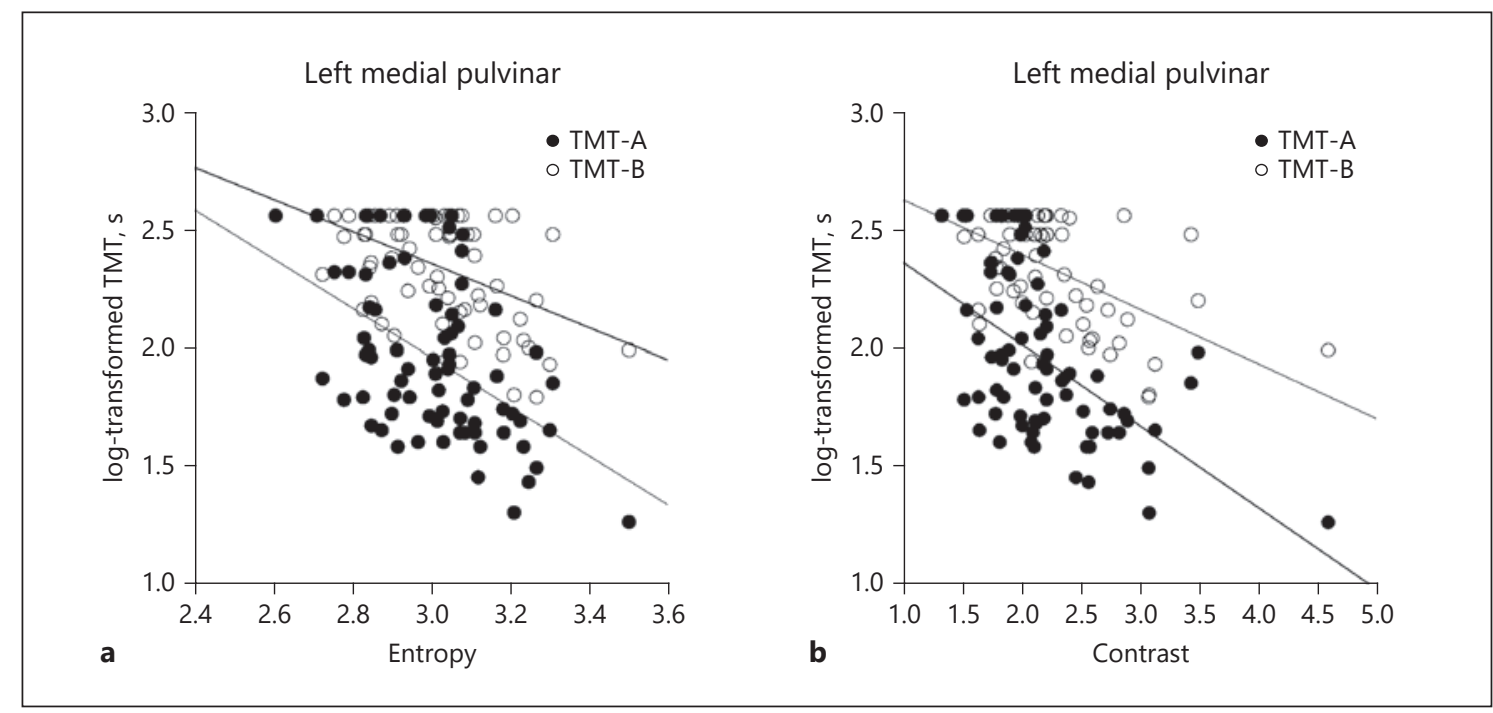

Fig. 1. Association between log-transformed TMT scores and texture features for all participants. a TMT-A: adjusted $R^{2}=0.231, \beta=-0.492, p<0.001$; TMT-B: adjusted $R^{2}=0.226, \beta=-0.487, p<0.001$. $\mathbf{b}$ TMT-A: adjusted $R^{2}=0.281, \beta=-0.539, p<0.001$; TMT-B: adjusted $R^{2}=0.290, \beta=-0.547, p<0.001$. ${ }^{*}$ Linear regression analysis.
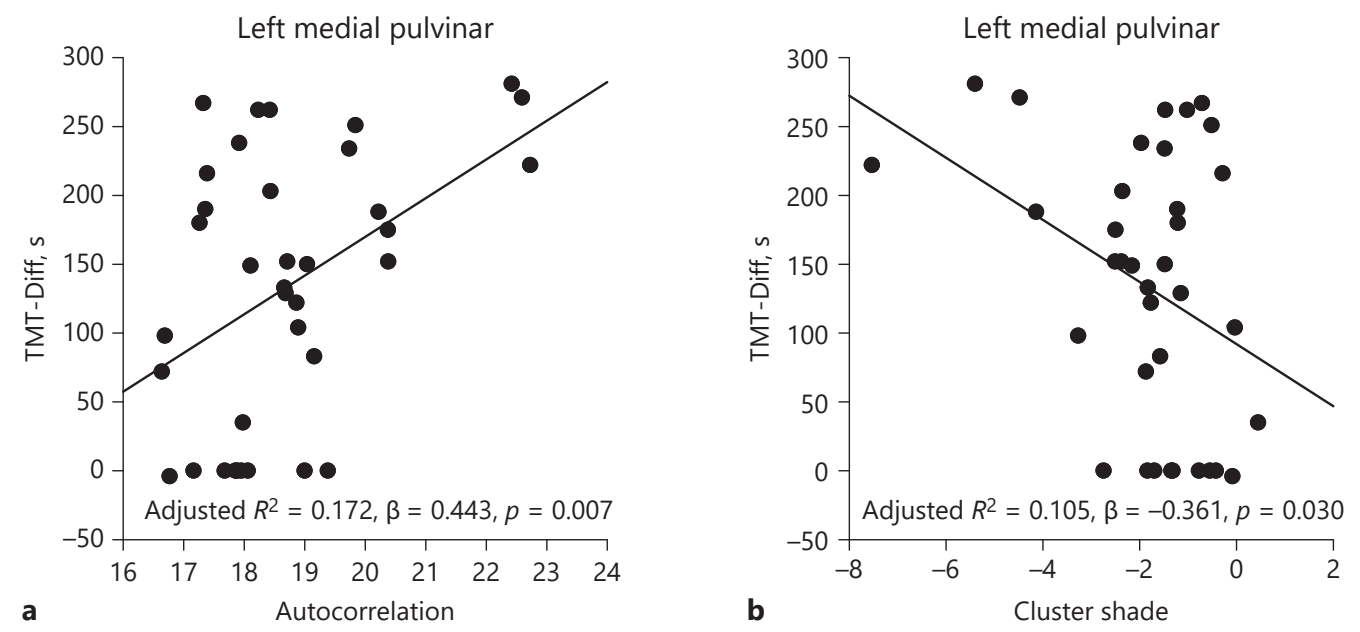

Fig. 2. Association between derived TMT scores and texture features for DLB. TMT-Diff = Trail Making Test-B minus Trail Making Test-A. * Linear regression analysis.

PuM may reflect pulvinar connections with asymmetrical neocortical regions involved in DLB. Although the texture changes in left PuM may be attributable to the increase of homogeneity due to neuronal losses, the volume of left PuM was comparable between the DLB patients and the controls. Since $95 \%$ of the DLB patients were in very mild or mild stage, the neuronal loss in the left PuM may have changed the texture but not yet the volume.

Pulvinar in Dementia with Lewy Bodies
We also found that the texture features of left PuM that were different between the DLB patients and the normal controls had higher correlation with the TMT-A and -B scores (Fig. 1) than the other neuropsychological test scores. TMT is a neuropsychological test [41] that is frequently used for screening multiple cognitive processes, such as attention, visual scanning, motoric speed, sequencing, shifting, and flexibility [42]. The TMT-A is more like- 
ly to associate with attention and the TMT-B is associated with executive function $[42,43]$. In linear regression analysis, entropy predicted $24.2 \%$ of the variance in log TMT-A scores and $23.7 \%$ of the variance in $\log$ TMT-B scores having a moderate correlation $(R=0.492$ and $R=0.487)$, while contrast predicted $29.0 \%$ of the variance in log TMT-A scores and $30.0 \%$ of the variance in log TMT-B scores presenting a moderate degree of correlation $(R=0.539$ and $R=0.547)$. However, the texture features explained less than or equal to $15.0 \%$ of the variance in MMSE, digit span tests and TMT-Diff having a weak correlation $(R<0.4)$. Within DLB, texture features were correlated with TMTDiff scores (Fig. 2). The TMT-Diff, which is the difference between the TMT-B and TMT-A, accounted for set-shifting executive function relatively independent of visual scanning and motor speed $[43,44]$. In the analysis, autocorrelation predicted $19.6 \%$ of the variance in TMT-Diff scores presenting a moderate degree of correlation $(R=$ 0.443 ), while cluster shade predicted $13.1 \%$ of the variance in TMT-Diff scores having moderate correlation $(R=$ 0.361). The PuM may be involved in executive control, which is important feature in early DLB $[2,45]$, mirroring the $\mathrm{SN}$ abnormalities. The $\mathrm{SN}$ initiates cognitive control as it modulates the switch between default mode network and central executive network [8]. For supporting cognitive flexibility, major areas of the SN play an important role: the anterior insula cortex initiates neural switching for accessing attention to salient stimulus, and the dorsal anterior cingulate cortex selects the appropriate response [46, 47]. Damage to the $\mathrm{SN}$ has inhibited attention and cognitive control $[8,48,49]$. The SN can correspond to the PuM because of anatomical interconnections $[14,35]$. Also, the $\mathrm{PuM}$ has been positively related to $\mathrm{SN}$ connectivity in frontotemporal dementia and in posterior cortical atrophy [16, 17]. Consequently, the PuM may represent dysfunction of executive control in DLB.

This study has several limitations. The sample size was small. Intra-scan intensity variation may have affected the estimation of the texture features.

\section{Conclusion}

This study showed that the texture of left PuM in DLB patients was different from that of normal controls and associated with executive function, which suggests that the left PuM may be impaired and contribute to the development of executive dysfunction in DLB from early stage.

\section{Statement of Ethics}

All patients (or their guardians) and control subjects were fully informed about the study, and written consent was obtained from them. The study was approved by the Institutional Review Board of the Seoul National University Bundang Hospital.

\section{Disclosure Statement}

The authors have no conflicts of interest to declare.

\section{Funding Sources}

This research was supported by a fund (2019-ER6201-00) by Research of Korea Centers for Disease Control and Prevention and partly supported by Institute for Information \& communications Technology Promotion (IITP) grant funded by the Korea government (MSIT) (2018-0-00861, Intelligent SW Technology Development for Medical Data Analysis).

\section{Author Contributions}

K.T. and K.W.K. contributed to the study concept and design, analysis and interpretation of data, and manuscript elaboration. S.L. and E.C. participated in the acquisition and analysis of data. S.W.S., D.J.O., W.M., H.S.K., S.B., J.B.B., J.W.H., and J.H.K. contributed to the acquisition of clinical and imaging data.

Published in Celebration of the 30th Anniversary of the inception of Dementia and Geriatric Cognitive Disorders 1990-2020.

\section{References}

1 Spillantini MG, Schmidt ML, Lee VM, Trojanowski JQ, Jakes R, Goedert M. a-synuclein in Lewy bodies. Nature. 1997 Aug;388(6645): $839-40$.

2 McKeith IG, Dickson DW, Lowe J, Emre M, O'Brien JT, Feldman H, et al.; Consortium on DLB. Diagnosis and management of dementia with Lewy bodies: third report of the DLB Consortium. Neurology. 2005 Dec;65(12): 1863-72.
3 Rongve A, Soennesyn H, Skogseth R, Oesterhus R, Hortobágyi T, Ballard C, et al. Cognitive decline in dementia with Lewy bodies: a 5 -year prospective cohort study. BMJ Open. 2016 Feb;6(2):e010357.

4 Petrova M, Pavlova R, Zhelev Y, Mehrabian S, Raycheva M, Traykov L. Investigation of neuropsychological characteristics of very mild and mild dementia with Lewy bodies. J Clin Exp Neuropsychol. 2016;38(3):354-60.
5 Ferman TJ, Smith GE, Kantarci K, Boeve BF, Pankratz VS, Dickson DW, et al. Nonamnestic mild cognitive impairment progresses to dementia with Lewy bodies. Neurology. 2013 Dec;81(23):2032-8.

6 Blanc F, Colloby SJ, Philippi N, de Pétigny X, Jung B, Demuynck C, et al. Cortical thickness in dementia with Lewy bodies and Alzheimer's disease: a comparison of prodromal and dementia stages. PLoS One. 2015 Jun;10(6):e0127396. 
7 Blanc F, Colloby SJ, Cretin B, de Sousa PL, Demuynck C, O'Brien JT, et al. Grey matter atrophy in prodromal stage of dementia with Lewy bodies and Alzheimer's disease. $\mathrm{Al}$ zheimers Res Ther. 2016 Jul;8(1):31.

8 Sridharan D, Levitin DJ, Menon V. A critical role for the right fronto-insular cortex in switching between central-executive and default-mode networks. Proc Natl Acad Sci USA. 2008 Aug;105(34):12569-74.

9 Lowther ER, O’Brien JT, Firbank MJ, Blamire AM. Lewy body compared with Alzheimer dementia is associated with decreased functional connectivity in resting state networks. Psychiatry Res. 2014 Sep;223(3):192-201.

10 Olszewski J. The thalamus of the Macaca Mulatta: An atlas for use with the stereotaxic instrument. Karger; 1952.

11 Bridge H, Leopold DA, Bourne JA. Adaptive pulvinar circuitry supports visual cognition. Trends Cogn Sci. 2016 Feb;20(2):146-57.

12 Ouhaz Z, Fleming H, Mitchell AS. Cognitive functions and neurodevelopmental disorders involving the prefrontal cortex and mediodorsal thalamus. Front Neurosci. 2018 Feb; 12:33.

13 Rosenberg DS, Mauguière F, Catenoix $\mathrm{H}$, Faillenot I, Magnin M. Reciprocal thalamocortical connectivity of the medial pulvinar: a depth stimulation and evoked potential study in human brain. Cereb Cortex. 2009 Jun; 19(6):1462-73.

14 Romanski LM, Giguere M, Bates JF, Goldman-Rakic PS. Topographic organization of medial pulvinar connections with the prefrontal cortex in the rhesus monkey. J Comp Neurol. 1997 Mar;379(3):313-32.

15 Homman-Ludiye J, Bourne JA. The medial pulvinar: function, origin and association with neurodevelopmental disorders. J Anat. 2019 Sep;235(3):507-20.

16 Lee SE, Khazenzon AM, Trujillo AJ, Guo CC, Yokoyama JS, Sha SJ, et al. Altered network connectivity in frontotemporal dementia with C9orf72 hexanucleotide repeat expansion. Brain. 2014 Nov; 137(Pt 11):3047-60.

17 Fredericks CA, Brown JA, Deng J, Kramer A, Ossenkoppele R, Rankin K, et al. Intrinsic connectivity networks in posterior cortical atrophy: A role for the pulvinar? Neuroimage Clin. 2019;21:101628.

18 Erskine D, Thomas AJ, Attems J, Taylor JP, McKeith IG, Morris CM, et al. Specific patterns of neuronal loss in the pulvinar nucleus in dementia with lewy bodies. Mov Disord. 2017 Mar;32(3):414-22.

19 Goubran M, Hammond RR, de Ribaupierre S, Burneo JG, Mirsattari S, Steven DA, et al. Magnetic resonance imaging and histology correlation in the neocortex in temporal lobe epilepsy. Ann Neurol. 2015 Feb;77(2):23750.

20 Gowland PA, Stevenson VL. T1: The Longitudinal Relaxation Time. In: Tofts P, editor. Quantitative MRI of the Brain. John Wiley \& Sons; 2003. pp. 111-41.
21 Han JW, Kim TH, Kwak KP, Kim K, Kim BJ, Kim SG, et al. Overview of the Korean Longitudinal Study on Cognitive Aging and Dementia. Psychiatry Investig. 2018 Aug;15(8): 767-74.

22 Yoo S-W, Kim Y-S, Noh J-S, Oh K-S, Kim C-H, NamKoong K, et al. Validity of Korean version of the mini-international neuropsychiatric interview. Anxiety and Mood. 2006; 2(1):50-5.

23 Lee JH, Lee KU, Lee DY, Kim KW, Jhoo JH, Kim JH, et al. Development of the Korean version of the Consortium to Establish a Registry for Alzheimer's Disease Assessment Packet (CERAD-K): clinical and neuropsychological assessment batteries. J Gerontol B Psychol Sci Soc Sci. 2002 Jan;57(1):47-53.

24 Morris JC. The Clinical Dementia Rating (CDR): current version and scoring rules. Neurology. 1993 Nov;43(11):2412-4.

25 Wechsler D. Wechsler memory scale-revised. New York: Psychological Corporation; 1987.

26 Fischl B, Salat DH, Busa E, Albert M, Dieterich $M$, Haselgrove C, et al. Whole brain segmentation: automated labeling of neuroanatomical structures in the human brain. Neuron. 2002 Jan;33(3):341-55.

27 Iglesias JE, Insausti R, Lerma-Usabiaga G, Bocchetta M, Van Leemput K, Greve DN, et al.; Alzheimer's Disease Neuroimaging Initiative. A probabilistic atlas of the human thalamic nuclei combining ex vivo MRI and histology. Neuroimage. 2018 Dec;183:314-26.

28 Haralick RM, Shanmugam K, Dinstein IH. Textural features for image classification. IEEE Trans Syst Man Cybern. 1973;SMC3(6):610-21.

29 Collewet G, Strzelecki M, Mariette F. Influence of MRI acquisition protocols and image intensity normalization methods on texture classification. Magn Reson Imaging. 2004 Jan; 22(1):81-91.

30 Mahmoud-Ghoneim D, Alkaabi MK, de Certaines JD, Goettsche FM. The impact of image dynamic range on texture classification of brain white matter. BMC Med Imaging. 2008 Dec;8(1):18.

31 Ortiz A, Palacio AA, Górriz JM, Ramírez J, Salas-González D. Segmentation of brain MRI using SOM-FCM-based method and 3D statistical descriptors. Comput Math Methods Med. 2013;2013.

32 Jones EG. The thalamus. Springer Science \& Business Media; 2012.

33 Alonso-Caneiro D, Szczesna-Iskander DH, Iskander DR, Read SA, Collins MJ. Application of texture analysis in tear film surface assessment based on videokeratoscopy. J Optom. 2013;6(4):185-93.

34 van Griethuysen JJ, Fedorov A, Parmar C, Hosny A, Aucoin N, Narayan V, et al. Computational radiomics system to decode the radiographic phenotype. Cancer Res. 2017 Nov; 77(21):e104-7.

35 Benarroch EE. Pulvinar: associative role in cortical function and clinical correlations. Neurology. 2015 Feb;84(7):738-47.
36 Marui W, Iseki E, Nakai T, Miura S, Kato M, Uéda K, et al. Progression and staging of Lewy pathology in brains from patients with dementia with Lewy bodies. J Neurol Sci. 2002 Mar;195(2):153-9.

37 Henderson MX, Cornblath EJ, Darwich A, Zhang B, Brown H, Gathagan RJ, et al. Spread of $\alpha$-synuclein pathology through the brain connectome is modulated by selective vulnerability and predicted by network analysis. Nat Neurosci. 2019 Aug;22(8):1248-57.

38 Ingelsson M. Alpha-synuclein oligomersneurotoxic molecules in parkinson's disease and other lewy body disorders. Front Neurosci. 2016 Sep;10:408.

39 Rockenstein E, Nuber S, Overk CR, Ubhi K, Mante M, Patrick C, et al. Accumulation of oligomer-prone $a$-synuclein exacerbates synaptic and neuronal degeneration in vivo. Brain. 2014 May;137(Pt 5):1496-513.

40 Diógenes MJ, Dias RB, Rombo DM, Vicente Miranda H, Maiolino F, Guerreiro P, et al. Extracellular alpha-synuclein oligomers modulate synaptic transmission and impair LTP via NMDA-receptor activation. J Neurosci. 2012 Aug;32(34):11750-62.

41 Rabin LA, Barr WB, Burton LA. Assessment practices of clinical neuropsychologists in the United States and Canada: a survey of INS, NAN, and APA Division 40 members. Arch Clin Neuropsychol. 2005 Jan;20(1):33-65.

42 Strauss E, Sherman EM, Spreen O. A compendium of neuropsychological tests: Administration, norms, and commentary. Oxford University Press; 2006.

43 Arbuthnott K, Frank J. Trail making test, part $B$ as a measure of executive control: validation using a set-switching paradigm. J Clin Exp Neuropsychol. 2000 Aug;22(4):518-28.

44 Corrigan JD, Hinkeldey NS. Relationships between parts A and B of the Trail Making Test. J Clin Psychol. 1987 Jul;43(4):402-9.

45 Collerton D, Burn D, McKeith I, O'Brien J. Systematic review and meta-analysis show that dementia with Lewy bodies is a visualperceptual and attentional-executive dementia. Dement Geriatr Cogn Disord. 2003;16(4): 229-37.

46 Seeley WW, Menon V, Schatzberg AF, Keller J, Glover GH, Kenna H, et al. Dissociable intrinsic connectivity networks for salience processing and executive control. J Neurosci. 2007 Feb;27(9):2349-56.

47 Dajani DR, Uddin LQ. Demystifying cognitive flexibility: implications for clinical and developmental neuroscience. Trends Neurosci. 2015 Sep;38(9):571-8.

48 Menon V, Uddin LQ. Saliency, switching, attention and control: a network model of insula function. Brain Struct Funct. 2010 Jun; 214(5-6):655-67.

49 Li LM, Violante IR, Leech R, Hampshire A, Opitz A, McArthur D, et al. Cognitive enhancement with Salience Network electrical stimulation is influenced by network structural connectivity. Neuroimage. 2019 Jan;185: 425-33. 